\title{
Erythropoietin promotes peripheral nerve regeneration in rats by upregulating expression of insulin-like growth factor-1
}

Wei Wang ${ }^{1}$, Dongsheng $\mathrm{Li}^{1}$, Qing $\mathrm{Li}^{2}$, Lei Wang ${ }^{1}$, Guang Bai ${ }^{1}$, Tao Yang ${ }^{1}$, Qiang $\mathrm{Li}^{1}$, Zhitu Zhu ${ }^{1}$, Hongzhi Sun ${ }^{1}$

${ }^{1}$ Department of General Surgery, The First Affiliated Hospital, Liaoning Medical University, Jinzhou, China

${ }^{2}$ Department of Renal Medicine, The Third Affiliated Hospital, Liaoning Medical University, Jinzhou, China

Submitted: 12 March 2013

Accepted: 30 April 2013

Arch Med Sci 2015; 11, 2: 433-437

DOI: 10.5114 /aoms.2015.50976

Copyright (c) 2015 Termedia \& Banach

\section{Abstract}

Introduction: Erythropoietin (EPO) has been shown to have beneficial effects on peripheral nerve damage, but its mechanism of action remains incompletely understood. In this study we hypothesized that EPO promotes peripheral nerve repair via neurotrophic factor upregulation.

Material and methods: Thirty adult male Wistar rats were employed to establish a sciatic nerve injury model. They were then randomly divided into two groups to be subjected to different treatment: $0.9 \%$ saline (group A) and $5000 \mathrm{U} / \mathrm{kg}$ EPO (group B). The walking behavior of rats was evaluated by footprint analysis, and the nerve regeneration was assessed by electron microscopy. The expression of insulin-like growth factor-1 (IGF-1) in the injured sciatic nerves was detected by immunohistochemical analysis.

Results: Compared to saline treatment, EPO treatment led to the growth of myelin sheath, the recovery of normal morphology of axons and Schwann cells, and higher density of myelinated nerve fibers. Erythropoietin treatment promoted the recovery of SFI in the injured sciatic nerves. In addition, EPO treatment led to increased IGF-1 expression in the injured sciatic nerves.

Conclusions: Erythropoietin may promote peripheral nerve repair in a rat model of sciatic nerve injury through the upregulation of IGF-1 expression. These findings reveal a novel mechanism underlying the neurotrophic effects of EPO.

Key words: erythropoietin, insulin-like growth factor-1, peripheral nerve repair, sciatic nerve.

\section{Introduction}

Peripheral nerves are frequently subjected to various forms of damage such as crushing, compression, stretching, avulsion and division. Due to the degeneration of motor neurons, the lack of a survival environment for Schwann cells, and the poor ability of the nerve to regenerate, peripheral nerve injuries are associated with major functional deficits and neuroma when severed axons are unable to reestablish continuity with the distal nerve [1]. Consequently, peripheral nerve trauma is a significant cause of morbidity and remains a great challenge for clinicians [2].

\author{
Corresponding author: \\ Hongzhi Sun \\ Department \\ of General Surgery \\ The First Affiliated Hospital \\ Liaoning Medical University \\ No. 2, 5th \\ Duan, People's Street \\ Guta District, Jinzhou, China, \\ 121001 \\ Phone: 864164197026 \\ Fax: 864164161152 \\ E-mail: zaishun5@yahoo.com
}


A promising therapeutic approach for peripheral nerve injury is to promote nerve regeneration, which includes the re-growth of injured axons as well as myelination, the restoration of synaptic connections and the recovery of physiological functions [3-5]. Erythropoietin (EPO) is originally identified as a renal cytokine that promotes hematopoiesis, but recent studies have shown that the administration of EPO enhanced axonal regrowth of peripheral nerve fibers and promoted functional recovery after peripheral nerve injury in rat models $[6,7]$. However, the mechanism of action of EPO in peripheral nerve repair remains incompletely understood.

As a new neurotrophic factor, insulin-like growth factor-1 (IGF-1) has been shown to play an important role in promoting axonal growth from neurons [8]. Based on the previous studies, we proposed that EPO could modulate the expression of IGF-1 to promote the repair of peripheral nerves after injury.

In this study, we employed the rat as a model of peripheral nerve injury and demonstrated that EPO upregulated the expression of IGF-1 and promoted the regeneration of myelinated nerve fibers and recovery after injury of the sciatic nerve.

\section{Material and methods}

\section{Animal models and experimental groups}

Thirty adult male Wistar rats ( 6 weeks old, 200 \pm 10 g) were provided by the Animal Center of Liaoning Medical University. The rats were randomly divided into 2 groups $(n=15)$ and anesthetized with an intraperitoneal injection of $10 \%$ chloral hydrate ( $3 \mathrm{ml} / \mathrm{kg}$ body weight), then shaved and washed with antiseptic solution before positioning for surgery. The left sciatic nerve and its two major branches were exposed through a gluteal muscle-splitting incision. A crush injury was created using a fine watchmaker forceps for $10 \mathrm{~s}$ on the peroneal nerve at $10 \mathrm{~mm}$ from the extensor digitorum longus muscle, and complete crush was confirmed by the presence of a translucent band across the nerve. The incision was then closed in layers (muscle and skin) with absorbable sutures. All operations were performed on the left limb, and the right limb served as a non-operated control. After the surgery, the rats were divided as follows: group A, $0.9 \%$ saline was injected into the injured site; and group B, EPO (5000 U/kg) was injected into the injured site. The injection was performed once daily for 5 weeks. At 1,3 and 5 weeks, 5 rats were randomly selected from each group for a sciatic functional index (SFI) test, then the rats were sacrificed and $1 \mathrm{~cm}$ of the left sciatic nerve was immediately taken from each rat as an experimental sample. In addition, $1 \mathrm{~cm}$ of the right sciatic nerve was immediately taken from each rat as an experimental sample.

\section{Sciatic functional index test}

At 1, 3 and 5 weeks after the treatment, the rats were put on a walking track $(15 \mathrm{~cm} \times 50 \mathrm{~cm})$ darkened at one end. White office paper cut to the appropriate dimensions was placed on the bottom of the track. The rat's hind limbs were dipped in Chinese ink, and the rat was permitted to walk down the track, leaving its hind foot prints on the paper. The SFI value was calculated based on the analysis of walking tracks as described previously [9]. Functional recovery was assessed by calculating the SFI ratio with the SFI value of 0 set as normal and the $\mathrm{SFI}$ value of -100 set as complete injury.

\section{Immunohistochemical analysis}

Immunohistochemical analysis of the sciatic nerves was performed as described previously [10]. Briefly, the sciatic nerves were fixed in 10\% formaldehyde and were paraffin wax-embedded. Subsequently, the tissues were cut into $5 \mu \mathrm{m}$ thick serial sections, which were washed carefully with phosphate buffered saline (PBS) three times. Next, dewaxed paraffin sections were treated with $3 \%$ hydrogen peroxide for 20 min to block endogenous peroxidases. The sections were blocked with $2 \%$ goat serum in PBS for $1 \mathrm{~h}$ at room temperature, incubated at $4^{\circ} \mathrm{C}$ overnight with antibody against IGF-1 (1 : 250 dilution, Santa Cruz Biotechnology, Santa Cruz, CA, USA), then subjected to immunohistochemical staining using a PV6000 kit (Bioss Inc. Beijing, China). Next, the sections were washed with PBS and developed with DAB for 5 min. Finally the sections were counterstained with hematoxylin and assessed by two independent investigators using light microscopy. The staining density was calculated based on the absorbance $(A)$ value of the nerves using an image analysis system.

\section{Electron microscopy}

The sciatic nerves were extracted from different groups of rats, fixed with osmium tetroxide, dehydrated with acetone, and sliced into $0.5 \mu \mathrm{m}$ ultra thin sections. The sections were dual stained with uranium acetate and lead citrate. The ultrastructure of the nerves was examined using a JEM 2000 EX transmission electron microscope (JEOL, Japan).

\section{Statistical analysis}

The data were expressed as mean \pm SD and analyzed by SPSS 13.0 software. Single factor analysis of variance was used for comparisons among groups. Value of $p<0.05$ was considered as statistically significant. 


\section{Results}

\section{EPO promotes the recovery of SFI in the injured sciatic nerves}

Walking track analysis demonstrated that 1 week after the injury, SFI values in group $A$ and $B$ showed no significant differences. However, 3 weeks after the injury, the SFI value was recovered as $-82.56 \pm 3.77$ in group $B$, significantly higher than in group A $(-95.12 \pm 3.36)(p<0.05)$. Furthermore, 5 weeks after the injury, the SFI value had recovered to $-63.50 \pm 4.27$ in group $B$, significantly higher than in group A $(-95.26 \pm 3.85)$ $(p<0.05)$ (Figure 1). These results demonstrated that EPO promoted the recovery of SFI in the injured sciatic nerves in a time-dependent manner.

\section{EPO promotes regeneration of the injured sciatic nerves}

Next we performed morphological analysis on the injured sciatic nerves in different groups. Electron microscopy analysis showed that in control sciatic nerves the morphologies of the axons and Schwann cells were normal (Figure 2 A). At 3 weeks after the injury, the myelin sheath was seriously damaged and many vacuoles were formed

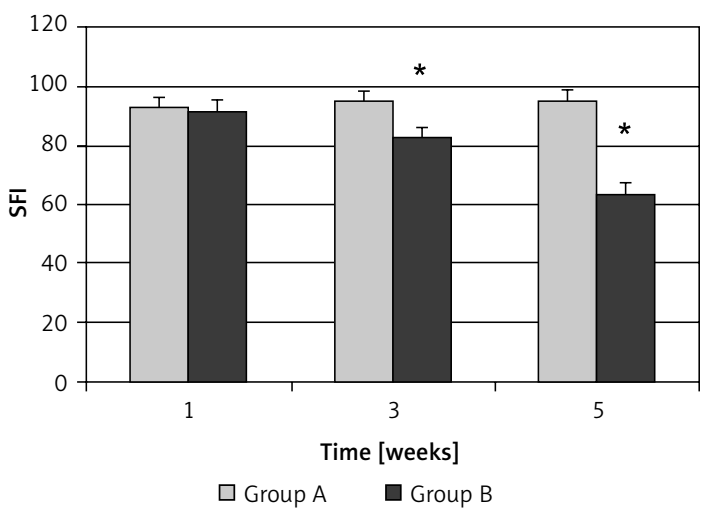

Figure 1. The SFI of the injured sciatic nerves in different groups. The SFI is expressed as $x \pm s(n=5)$. Group A, $0.9 \%$ saline treatment; group B, EPO (5000 U/kg) treatment

${ }^{\star} P<0.05$ vs. group $A$ at 3 weeks and 5 weeks, respectively. in the middle of the axons in group A (Figure $2 \mathrm{~B}$ ). However, the damaged myelin sheath grew, and the axons and Schwann cells exhibited normal morphology after EPO treatment for 3 weeks in group B (Figure $2 \mathrm{C}$ ). Furthermore, we analyzed the density of the myelinated nerve fibers in different groups and observed that EPO treatment significantly increased the density of the myelinated nerve fibers at 3 weeks and 5 weeks in group B compared to group A (Figure 3 ). Taken together, these results demonstrate that EPO promotes regeneration of the injured sciatic nerves.

\section{EPO increases the expression of IGF-1 \\ in the injured sciatic nerves}

By immunohistochemical staining, the expression of IGF-1 in the injured sciatic nerves was detected. The results showed that 1 week after the injury, IGF-1 expression was strong in both group $A$ and $B$ (Figures $4 A, D$ ), suggesting that endogenous IGF-1 is induced in response to the injury. However, IGF-1 expression was gradually decreased at 3 weeks and 5 weeks after the injury in group $\mathrm{A}$, while it was still maintained at a higher level at 3 weeks and 5 weeks after the injury in group B compared to group A (Figures 4 B, C, E, F).

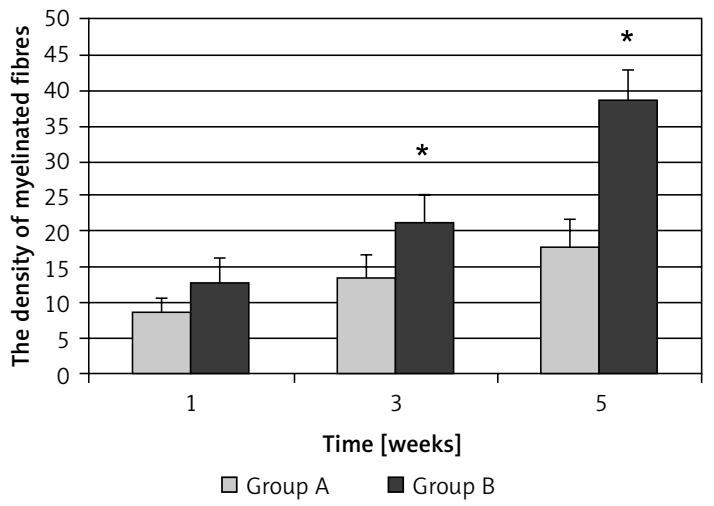

Figure 3. Density of myelinated fibers in the injured sciatic nerves in different groups. Data are expressed as $x \pm \mathrm{s}\left(10^{3} / \mathrm{mm}^{2}\right)(n=5)$. Group A, $0.9 \%$ saline treatment; group B, EPO (5000 U/kg) treatment ${ }^{*} P<0.05$ vs. group $A$ at 3 weeks and 5 weeks, respectively.
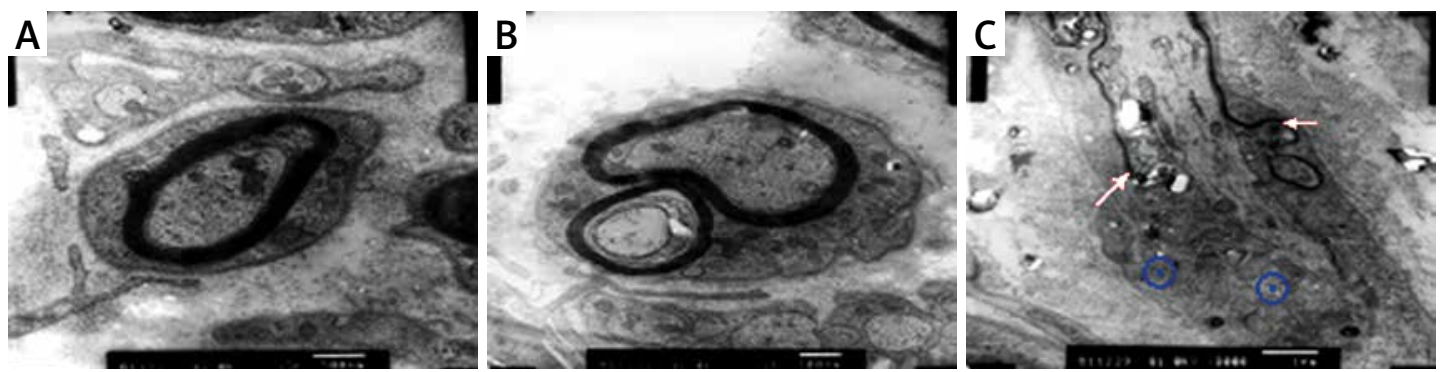

Figure 2. Morphological analysis of the injured sciatic nerves in different groups. The nerves were examined by electron microscopy. Representative images are shown. A - Uninjured sciatic nerves; B - injured sciatic nerves 3 weeks after $0.9 \%$ saline treatment; $\mathbf{C}$ - injured sciatic nerves 3 weeks after EPO (5000 U/kg) treatment. Arrows indicate the re-growth of myelin sheath, and blue circles indicate Schwann cells. Magnification: 5,000× 

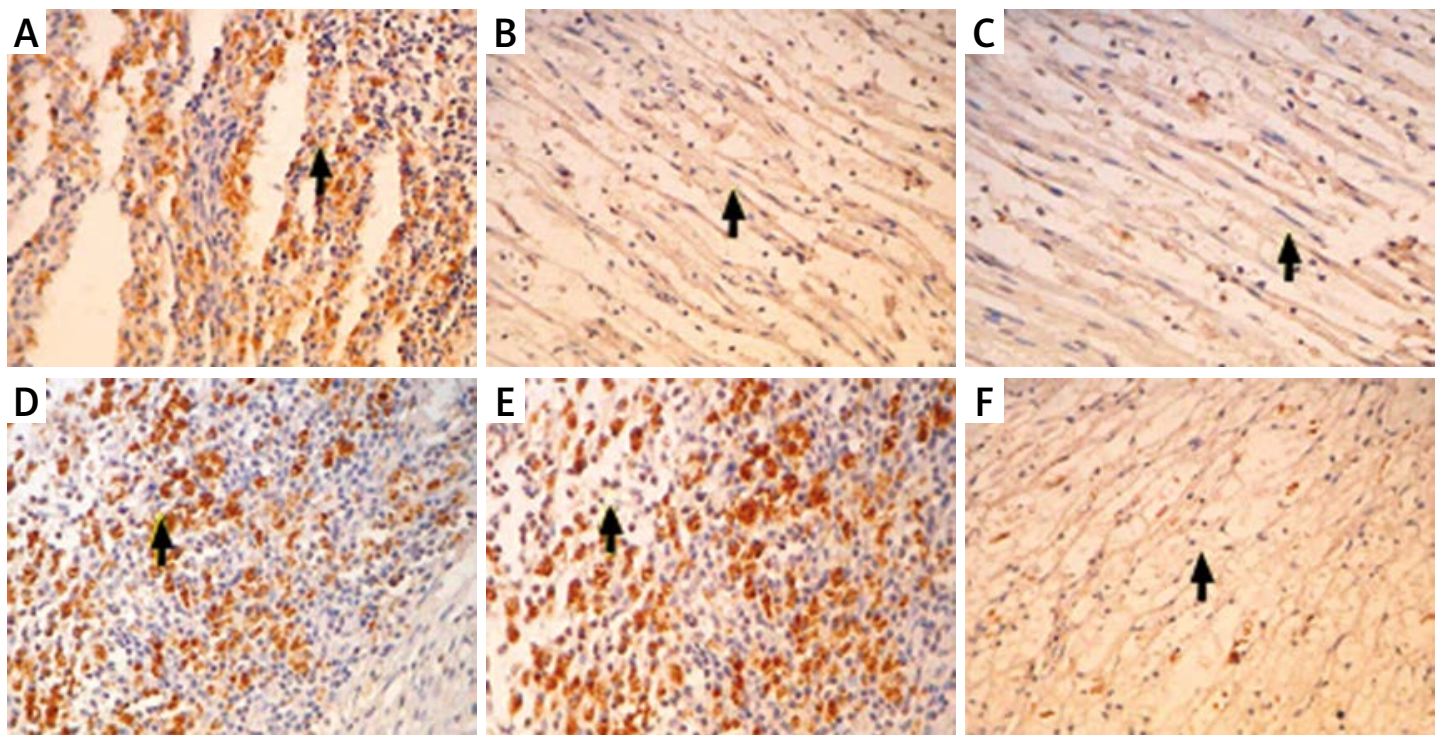

Figure 4. Immunohistochemical staining of IGF-1 in the sciatic nerves in different groups. The nerves were subjected to immunohistochemical staining with IGF-1 antibody. Representative images are shown. A - Injured sciatic nerves 1 week after $0.9 \%$ saline treatment; $\mathbf{B}$ - injured sciatic nerves 3 weeks after $0.9 \%$ saline treatment; $\mathbf{C}$ - injured sciatic nerves 5 weeks after $0.9 \%$ saline treatment; D - injured sciatic nerves 1 week after EPO (5000 U/kg) treatment; $\mathbf{E}$ - injured sciatic nerves 3 weeks after EPO (5000 U/kg) treatment; $\mathbf{F}$ - injured sciatic nerves 5 weeks after EPO (5000 U/kg) treatment. Magnification: 200x

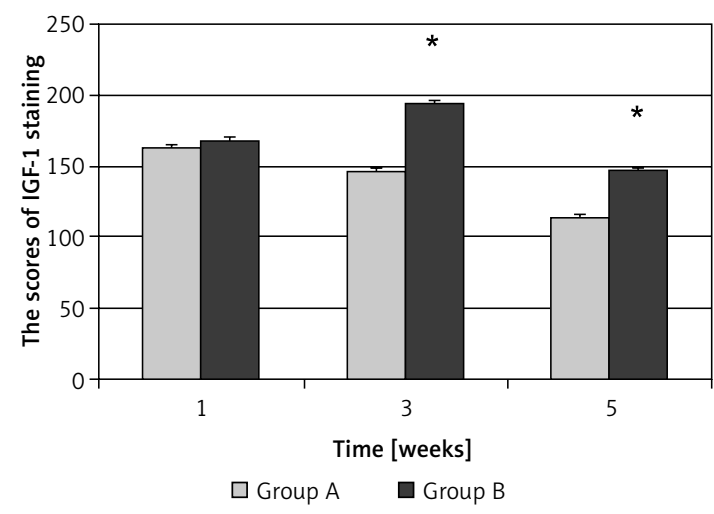

Figure 5. Scores of immunohistochemical staining of IGF-1 in the sciatic nerves in different groups. The score is expressed as $x \pm s$ (A value) $(n=5)$. Group $A, 0.9 \%$ saline treatment; group $B$, EPO (5000 U/kg) treatment

${ }^{\star} P<0.05$ vs. group $A$ at 3 weeks and 5 weeks, respectively.

Quantitative analysis of IGF-1 staining showed significant differences between Group A and group $B$ at 3 weeks and 5 weeks (Figure 5). These results indicate that EPO treatment increased IGF-1 expression in the injured sciatic nerves, which may explain the beneficial effects of EPO on the structural and functional recovery of sciatic nerves in response to the injury.

\section{Discussion}

As early as in 1993, it was shown that the administration of EPO promoted the survival of septal cholinergic neurons in adult rats which had undergone fimbria-fornix transactions, suggest- ing the neurotrophic effects of EPO [11]. Subsequently, a variety of experimental studies have confirmed the neurotrophic effects of EPO $[4,6$, 7]. Nevertheless, the mechanisms underlying the neurotrophic effects of EPO remain elusive.

In this study we employed a variety of approaches to characterize the morphological and functional aspects of rat sciatic nerves subjected to injury and treatment by EPO. Morphologically, by electron microscopy we observed that compared to saline treatment, EPO treatment led to the growth of myelin sheath, the recovery of normal morphology of axons and Schwann cells, and higher density of myelinated nerve fibers. Functionally, we demonstrated that EPO treatment promoted the recovery of SFI in the injured sciatic nerves. Taken together, these results suggest that EPO promotes the regeneration of myelinated nerve fibers. Mechanistically, we demonstrated that EPO treatment increased the expression of IGF-1 in the injured sciatic nerves at the protein level. Based on our results, we postulate that EPO promotes the regeneration and functional recovery of the injured sciatic nerves at least partially via the upregulation of IGF-1 expression. However, in the present study we did not examine the effects of EPO treatment on the expression of other neurotrophic factors, and further studies are important to identify other neurotrophic factors that may mediate the neurotrophic effects of EPO on peripheral nerves.

In conclusion, in this study we provide evidence that EPO promotes peripheral nerve repair in a rat model of sciatic nerve injury via the upregulation 
of IGF-1 expression. Our results shed new light on the neurotrophic effects of EPO and support the clinical application of EPO in the treatment of peripheral nerve injury.

\section{Acknowledgments}

Hongzhi Sun and Dongsheng Li contributed equally.

This study was supported by the following grants: Fund from Department of Science and Technology of Liaoning Province (No. 2013225305) and Fund from Liaoning Medical University (No. Y2011B06).

\section{Conflict of interest}

The authors declare no conflict of interest.

\section{References}

1. Deumens R, Bozkurt A, Meek MF, et al. Repairing injured peripheral nerves: bridging the gap. Prog Neurobiol 2010; 92: 245-76.

2. Robinson LR. Traumatic injury to peripheral nerves. Muscle Nerve 2000; 23: 863-73.

3. Yu W, Wang J, Yin J. Platelet-rich plasma: a promising product for treatment of peripheral nerve regeneration after nerve injury. Int J Neurosci 2011; 121: 176-80.

4. Nagańska E, Taraszewska A, Matyja E, Grieb P, Rafałowska J. Neuroprotective effect of erythropoietin in amyotrophic lateral sclerosis (ALS) model in vitro. Ultrastructural study. Folia Neuropathol 2010; 48: 35-44.

5. Schaller BJ. The role of endothelin in stroke: experimental data and underlying pathophysiology. Arch Med Sci 2006; 2: 146-58.

6. Zhang W, Sun B, Yu Z, An J, Liu Q, Ren T. High dose erythropoietin promotes functional recovery of rats following facial nerve crush. J Clin Neurosci 2009; 16: 554-6.

7. Yin ZS, Zhang H, Bo W, Gao W. Erythropoietin promotes functional recovery and enhances nerve regeneration after peripheral nerve injury in rats. Am J Neuroradiol 2010; 31: 509-15.

8. Xiang Y, Ding N, Xing Z, Zhang W, Liu H, Li Z. Insulin-like growth factor-1 regulates neurite outgrowth and neuronal migration from organotypic cultured dorsal root ganglion. Int J Neurosci 2011; 121: 101-6.

9. Liu GB, Cheng YX, Feng YK, et al. Adipose-derived stem cells promote peripheral nerve repair. Arch Med Sci 2011; 7: 592-6.

10. Sun H, Yang T, Li Q, et al. Dexamethasone and vitamin $B(12)$ synergistically promote peripheral nerve regeneration in rats by upregulating the expression of brain-derived neurotrophic factor. Arch Med Sci 2012; 8: 924-30.

11. Konishi $\mathrm{Y}$, Chui DH, Hirose $\mathrm{H}$, et al. Trophic effect of erythropoietin and other hematopoietic factors on central cholinergic neurons in vitro and in vivo. Brain Res 1993; 609: 29-35. 